\title{
Epstein-Barr virus associated lymphoproliferative disorder with fatal involvement of the gastrointestinal tract in an infant
}

\author{
L X Pan, P Ramani, T C Diss, L N Liang, P G Isaacson
}

\begin{abstract}
A case of fatal Epstein-Barr virus (EBV) associated lymphoproliferative disorder is reported in an 11 month old female. Heavy infiltrates of CD20+ and EBV EBER mRNA expressing lymphoid blasts were found to cause a series of ulcers along the entire length of the gastrointestinal tract and there was an ileal perforation. Similar infiltrates were also found in lymph nodes, spleen, and liver. Although blood phenotypic analysis performed shortly before her death revealed a severe decrease in $T$ lymphocytes, neither the patient nor other members of her family had a history of primary or secondary immunodeficiency. EBV infection is common in children. However, such a fatal infection of the virus has not apparently been described previously in infants without pre-established immunodeficiency.

(f Clin Pathol 1995;48:390-392)
\end{abstract}

Keywords: EBV, gastrointestinal tract, lymphoproliferation, infant.

Epstein-Barr virus (EBV) infection occurs commonly in children. In infants, such infection is relatively rare and usually silent. ${ }^{1}$

Accepted for publication 26 September 1994

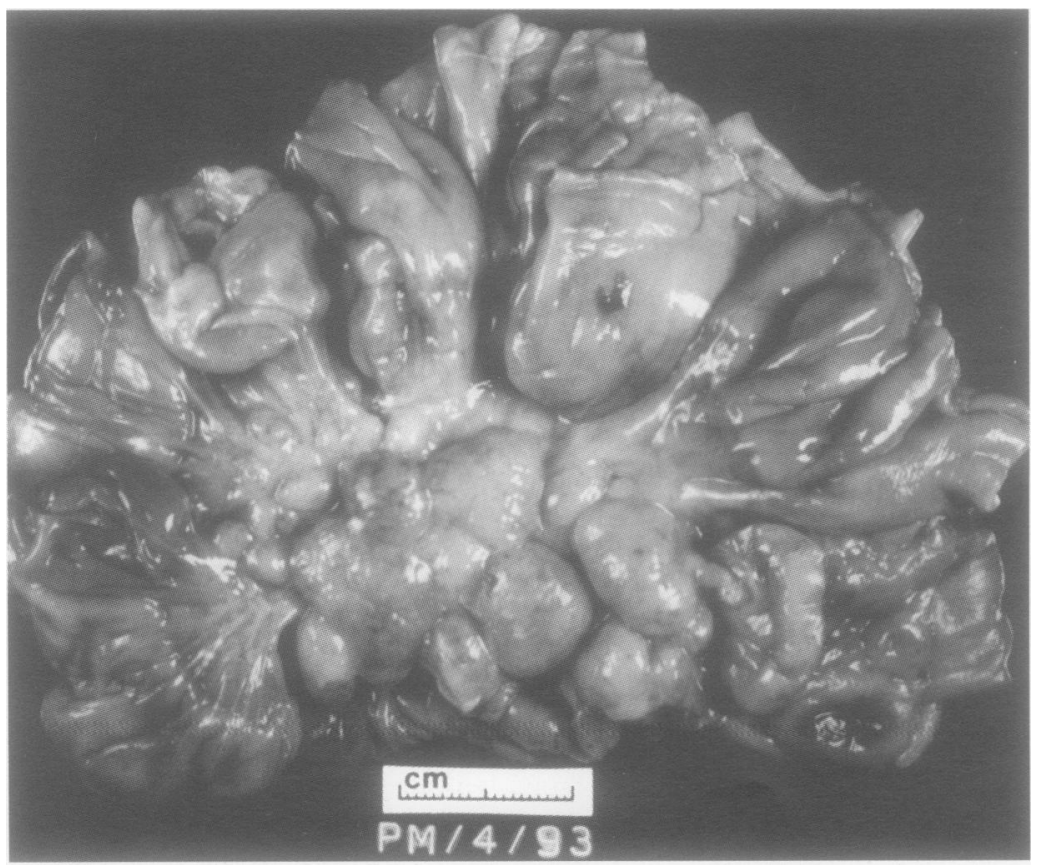

Figure 1. EBV infected gut with a perforation in the terminal ileum and enlarged mesenteric lymph nodes.
In older children the infection tends to be symptomatic as a result of infectious mononucleosis, a benign self limiting lymphoproliferation. In patients with primary and secondary immunodeficiencies, EBV infection may lead to fatal lymphoproliferative diseases, ranging from extensive polyclonal diffuse lymphoid hyperplasia to monoclonal lymphomas. ${ }^{12}$

In this report, we describe an 11 month old girl who, despite having no history of immunodeficiency, died from severe haemorrhage, ulceration, and perforation of the gastrointestinal tract caused by extensive EBV associated oligoclonal lymphoproliferatiion.

\section{Case report}

The patient, an 11 month old female, presented with fever, diarrhoea, and generalised lymphadenopathy over two weeks. Viral serology tests on admission showed that serum IgG to EBV nuclear antigen (EBNA) was positive, while EBV IgM to EBNA and EBV capsid antigen (VCA) were negative. Cytomegalovirus latex test was also negative. Total white blood cell count was $6.6 \times 10^{3} / \mathrm{mm}^{3}$ and lymphocyte count was $2.05 \times 10^{3} / \mathrm{mm}^{3}$. The child developed persistent abdominal distension, and coffee ground nasogastric aspirates indicated haemorrhage. Chest $x$ ray and abdominal $x$ ray showed free subdiaphragmatic air. The patient died two weeks after presentation following severe peritonitis. Flow cytometric analysis of blood lymphocytes performed 24 hours before the death showed CD2-14\%, CD3-9\%, CD4$1 \%$, CD8-15\%, CD16/56-3\%, and CD19$71 \%$, indicating a decrease in $\mathrm{T}$ lymphocytes, especially $\mathrm{T} 4$ (helper/inducer) and an increase in B lymphocytes.

The patient was noted since birth to have several dysmorphic features such as a triangular face, micrognathia, low set ears, and hypotonia. Chromosomal studies showed normal 46XX karyotype. No specific syndrome could be identified. Her elder sister (age three years) had similar dysmorphic features, but neither the patient nor other members of her family had a history of primary or secondary immunodeficiency.

Necropsy showed, in addition to generalised lymphadenopathy, a series of ulcers along the whole length of the gastrointestinal tract, with a perforation of the ileum (fig 1). There was associated peritonitis with $100 \mathrm{ml}$ of purulent fluid in the abdominal cavity. The tonsils, spleen, and liver were not enlarged. There was 


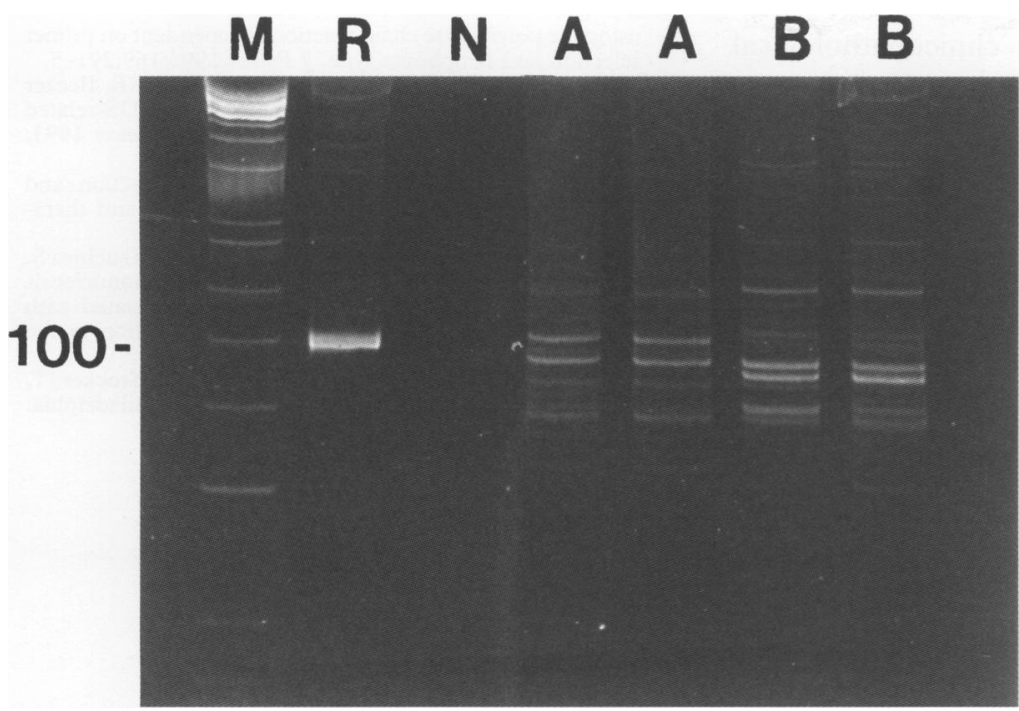

Figure 2 Polymerase chain reaction (PCR) analysis of rearranged Ig VH gene shows oligoclonal patterns of the lymphoid lesions at two different sites of the gut $(A$ and $B$; duplicated PCR reactions). $M=P h i-X$ HinfI marker; $R=$ Raji cell line monoclonal control; $N=$ negative control without DNA template.

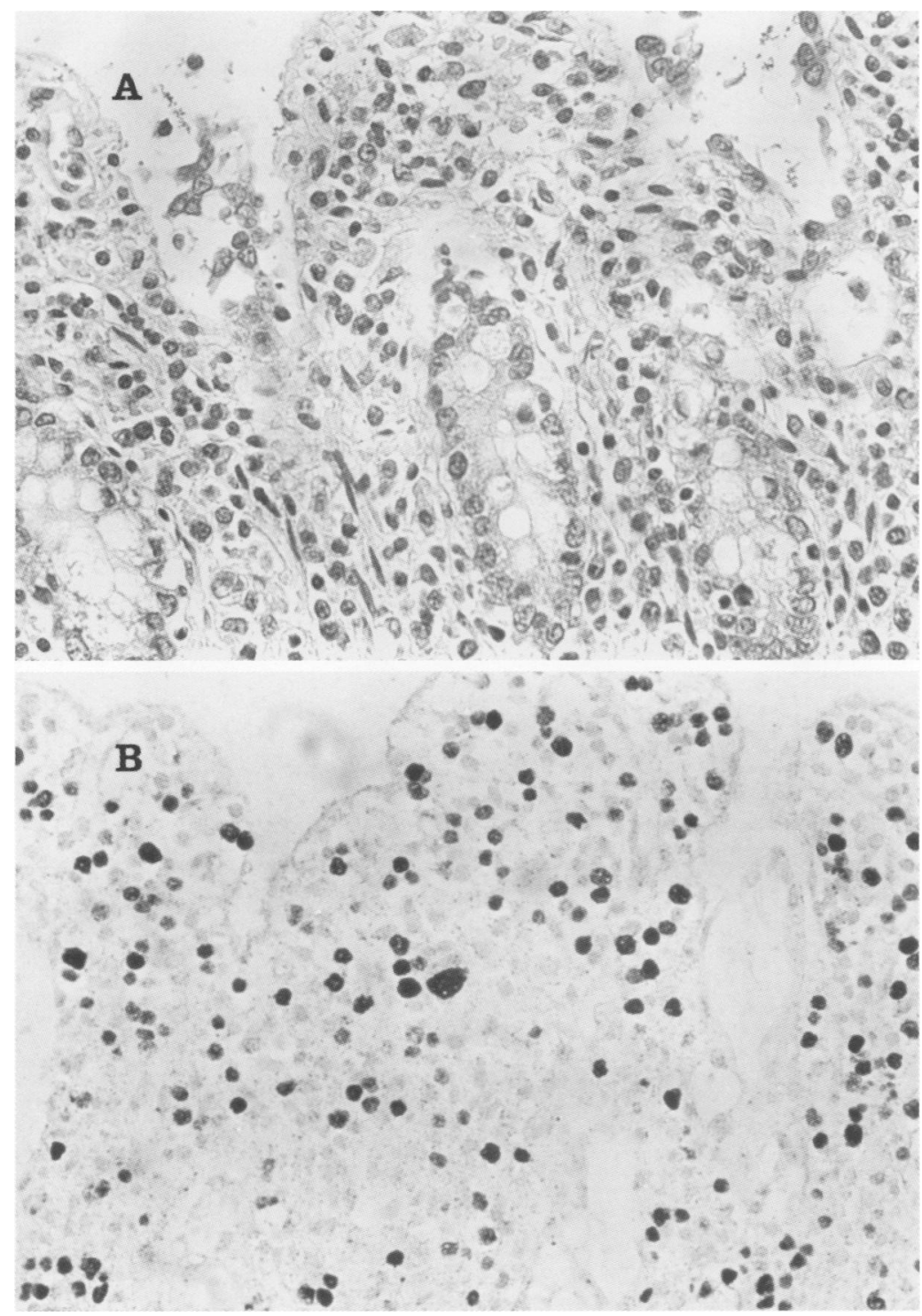

Figure 3 A: Haematoxylin and eosin stained tissue section showing heavy infiltration of gut mucosa by lymphoid blasts. B: In situ hybridisation for EBV EBER $m R N A$ showing strong signal in blast cells.
Histological sections from the ulcers sampled from multiple sites of the gut showed a dominant population of monomorphic lymphoid blasts accompanied by few plasmacytoid cells and small lymphocytes in the mucosa. In some areas, this infiltrate extended through the muscle layers into the serosa. In the enlarged lymph nodes from different sites of the body, these cells caused architectural effacement. Similar infiltration was also observed in the portal tracts of the liver, white pulp of the spleen, and perivascular sites of the kidney and lung.

The infiltrating lymphoid blasts were predominantly $\mathrm{CD} 20+$ and showed focal evidence of light chain restriction but were mostly polytypic. EBV latent membrane protein (LMP) was weakly positive in some of these transformed cells. Polymerase chain reaction (PCR) for detection of immunoglobulin gene rearrangement ${ }^{3}$ revealed an oligoclonal pattern which varied in different sites of disease (fig 2). In situ hybridisation for EBV EBER $\mathrm{mRNA}^{4}$ showed a strong signal in virtually all the transformed blasts from lymphoid lesions of various organs (fig 3).

\section{Discussion}

The clinical features of the patient indicated an acute infection with severe involvement of the gastrointestinal tract. The results of the serologic tests after admission (IgM to VCA and EBNA negative and IgG to EBNA positive) suggested past infection with EBV. ${ }^{5}$ It is possible that the acute phase of this disease resulted from reactivation of past $E B V$ infection.

Fatal EBV associated polyclonal lymphoproliferative disorders have been described mainly in patients with immunodeficiencies, such as patients with $\mathrm{X}$ linked lymphoproliferative syndrome (XLPS), severe combined immunodeficiency (SCID), or after organ transplantation. ${ }^{12}$ In this patient, phenotypic analysis performed shortly before death showed a marked decrease in $\mathrm{T}$ lymphocytes. However, the lack of recurrent infections and family history of primary or secondary immunodeficiencies does not support a pre-existing immunodeficiency. We cannot exclude the possibility that the decrease in $\mathrm{T}$ lymphocytes in our patient represents a response secondary to overwhelming virus infection and terminal illness. ${ }^{67}$

The diagnostic problems presented by EBV infection continue to be a concern for pathologists. In this case, features such as effacement of architecture of the lymphoid organs and the dense and monomorphous infiltrate composed predominantly of transformed lymphoid blasts made it difficult to distinguish the disease from high grade B cell lymphoma. Using immunohistochemistry and PCR we were able to elucidate the oligoclonal nature of this fatal lymphoproliferation. In situ hybridisation enabled us to show that EBV infection was the cause of the disease. Therefore, to avoid an erroneous diagnosis of malignancy in similar lymphoproliferative disorders, it is no evidence of bronchopneumonia or skin rash. No gross congenital defects were noted in the internal organs. 
essential to correlate the clinicopathological findings with molecular results.

1 Schuster V, Kreth HW. Epstein-Barr virus infection and associated diseases in children. I. Pathogenesis, epidemiology and clinical aspects. Eur $\mathcal{f}$ Pediatr 1992;151: 718-25.

2 Weisenburger DD, Purtilo DT. Failure in immunological control of the virus infection: fatal infectious mononucleosis. In: Epstein MA, Achong BG eds. The EpsteinBarr virus: recent advances. London: Heinemann, 1986. 127-61.

3 Diss TC, Peng HZ, Wotherspoon AC, Isaacson PG, Pan L. Detection of monoclonality in low-grade B-cell lymphomas using the polymerase chain reaction is dependent on primer selection and lymphoma type. $\mathcal{F}$ Pathol 1993;169:291-5.

4 MacMahon EM, Glass JD, Hayward SD, Mann RB, Becke

PS, Charache P, et al. Epstein-Barr virus in AIDS-related PS, Charache P, et al. Epstein-Barr virus in AIOS-related primary central nervous system lymphoma. Lancet 1991;

5 Schuster V, Kreth HW. Epstein-Barr virus infection and associated diseases in children. II. Diagnostic and therapeutic strategies. Eur $\mathcal{f}$ Pediatr 1992;151:794-8.

6 Tazawa Y, Nishinomiya F, Noguchi H, Takada G, Tsuchiya S, Sumazaki $R$, et al. A case of fatal infectious mononucleosis presenting with fulminant hepatic failure associated with an extensive CD8-positive lymphocyte infiltration in the liver. Hum Pathol 1993;24:1135-9.

7 Abramowsky C. Immunodeficiency disorders. In: Stocker JT, Dehner LP, eds. Pediatric pathology, Vol I. Philadelphia: Dehner LP, eds. Pediatric
Lippincott, 1992:372-4. 